JOURNAL OF SCIENTIFIC PERSPECTIVES
E-ISSN: $2587-3008$
ACADEMY
Jultp://ratingacademy.com.tr/journals/index.php/jsp/

\title{
THE EFFECT OF HOME VISITS BY NURSES ON THE PHYSICAL AND PSYCHOSOCIAL HEALTH OF THE ELDERLY: STUDY PROTOCOL FOR A SYSTEMATIC REVIEW AND META-ANALYSIS
}

\author{
Dr. Emine ERGIN** \\ Prof. Dr. Belgin AKIN* \\ Assoc. Prof. Dr. Deniz TANYER* \\ *Selcuk University, Health Science Faculty, Department of Public Health Nursing, Konya,Turkey, \\ E-mail: eminesariselcuk@gmail.com
}

\begin{tabular}{|c|c|}
\hline ARTICLE INFO & $\overline{\text { ABSTRACT }}$ \\
\hline $\begin{array}{l}\text { Article History: } \\
\text { Received: } 03 \text { May } 2018 \\
\text { Accepted: 09 July } 2018\end{array}$ & $\begin{array}{l}\text { One of the most significant global demographic tendencies is the rapidly aging } \\
\text { world population which will soon be a global health problem. Community health } \\
\text { nurses can easily address, observe the risk factors of the elderly's health and } \\
\text { health-promoting hehaviors during home visits Home visits reduce the mortality }\end{array}$ \\
\hline $\begin{array}{l}\text { Keywords: elderly, home } \\
\text { visit, meta-analysis. nursing, } \\
\text { systematic review. }\end{array}$ & $\begin{array}{l}\text { and hospitalization rates of the elderly and enable taking measures against many } \\
\text { risky situations. Scientific evidence exists for the fact that more frequent home } \\
\text { visits reduce mortality and hospitalization. The objective of systematic review } \\
\text { and meta analysis is to identify and synthesize the evidence on the effectiveness }\end{array}$ \\
\hline $\begin{array}{l}\text { DOI: } \\
\text { 10.26900/jsp.2018342248 }\end{array}$ & $\begin{array}{l}\text { of home visits by nurses on the physical and psychosocial health of the elderly. } \\
\text { Systematic review and meta analysis, study design was used. The included } \\
\text { studies design of randomized controlled, quasi-experimental and observational } \\
\text { (cohort, case-control, cross-sectional), Turkish and English, between December, } \\
2004 \text { and December } 2016 \text { (but not finished, ongoing) including a home visit } \\
\text { attempt and health outcomes will include physical and psychosocial health. } \\
\text { Medline (6860 articles), Web of Sciences (1933 articles), Pubmed (1551 } \\
\text { articles), Science Direct (1021 articles), Cinahl Plus with Full Text (1020 } \\
\text { articles), Cochrane (501 articles) Tubitak (98 articles), and Yök (126 articles) } \\
\text { were searched among a total of } 13.110 \text { abstract databases. Scientific evidence } \\
\text { exists for the fact that more frequent home visits reduce mortality and } \\
\text { hospitalization.The results of this study will help to identify and synthesize the } \\
\text { evidence on the effectiveness of home visits by nurses on the health of the elderly. }\end{array}$ \\
\hline
\end{tabular}

\section{INTRODUCTION}

One of the most significant global demographic tendencies is the rapidly aging world population which will soon be a global health problem (WPA, 2015; WPP, 2012). Thanks to the developments in health and socioeconomics, mortality and morbidity rates have fallen in both contagious diseases due to infectious conditions and in non-contagious diseases. When these demographic and epidemiological changes are integrated with rapid urbanism, modernization, globalization, risk factors, and the changes in life styles, chronic cases become increasingly important. Effective strategies are required for health systems in order to expand 
health services and meet the needs of elderly individuals aged 65 and above (Chatterji et al., 2015).

Along with the increase in elderly population, inequality in income distribution and insufficient access to health and social support services has increased, causing disease burden and global health risks. These health risks are associated with insufficient access to health services and poverty in most developing countries. For example, the epidemiological profiles are directed by a number of factors such as rapid urbanization, changing nutrition habits, or physical activity; however, valid and effective outcomes are obtained in developed countries (Steptoe et al., 2015; Suzman et al., 2015). The increasing percentage of the elderly population in the total population has brought health, environmental, economic and social problems. Today, mostly women and young girls are responsible for giving care to elderly individuals. Changes in family structure, transition from extended family type to nuclear family type and women's involvement in work life have increased the need of the elderly for institutional care (Tezcan and Seçkiner, 2012).

The success of protective and promotive efforts for elderly health depends on elderly health policies developed considering the needs of elderly individuals. Policies to be developed should be community-based and care-oriented. They should not include medical care alone. Community-based care covers maintaining and promoting health, and providing health and social services for elderly individuals in their own living areas to prevent or delay diseases and disabilities or to minimize their effects (Tarricone and Tsouros, 2008). Methods such as home visits by nurses are required for addressing elderly health problems. Home visits include a series of care at different levels: primary (health education, reduction of the risk, safety, protection and promotion of health), secondary (early diagnosis), and tertiary (adaptation to new conditions and rehabilitation). In addition, these home visits should focus on various subjects such as physical activity, drug compliance and environment (Gillespie et al., 2011).

It is not inevitable for elderly people to suffer in old age due to deteriorated health status or disability. Many elderly individuals want to live as long as possible in their own home, independently and in good health. One reason for this wish is the costs of institutional care in hospitals or nursing homes. Considering their wishes, policy-makers have made it a social and economic priority that elderly people receive care and treatment in their own homes to help protecting them from diseases and disabilities and promoting their health. Elderly people's social and economic difficulties and disability both cause them to feel more independent in their own home. Home visits for elderly individuals can be used to achieve these purposes more easily (Gillespie et al., 2011;Grady,2011; Steptoe et al., 2015; Suzman et al., 2015).

Nurses and scientists working in nursing have always been leaders in elderly care, and they will have a critical role in addressing elderly care problems in the future. They will continue to find scientific evidence to improve clinical care and enhance the quality of life of the aging population (Gillespie et al., 2011). Nurses provide healthcare for elderly individuals in first step health institutions, within the community, in hospitals, in nursing homes and in palliative care centers. Community health nurses can easily address and observe the risk factors of the elderly's health and health-promoting behaviors during home visits (Gillespie et al., 2009).

Home visits reduce the mortality and hospitalization rates of the elderly and enable taking measures against many risky situations such as falling. Based on the studies analyzing the lifelong costs of nursing homes, home visits with frequent follow-ups are recommended 
for each individual older than 40 years old (Stuck et al., 2002). Home visits can yield more cost-effective outcomes compared to long-term institutional care ( Chatterji etl al., 2015; Steptoe et al., 2015; Stuck et al., 2002). Scientific evidence exists for the fact that more frequent home visits reduce mortality and hospitalization, particularly for younger elderly individuals (aged 60). However, studies analyzing the effectiveness of home visit programs for elderly people may have conflicting results (Gillespie et al., 2009; Stuck et al., 2002; Van Haastregt et al., 2000).

Researchers have reported that a single study cannot be adequate when conflicting results are seen in the solution of a problem and recommended that the results of independent studies on the same subject be synthesized (Borenstein et al., 2009; Hadich, 2010). These studies consist of meta-analyses providing high-level evidence on the effective use of time and costs. This method assesses the inconsistencies in the literature and analyzes their causes, increasing the estimation power of the studies, obtaining cost-effective results and introducing new approaches that can be analyzed in subsequent studies (Haidich, 2010).

In conclusion, cost-effective (low cost) solutions should be developed to improve the functional conditions of elderly people and increase the effectiveness of interventions (Chatterji et al., 2015). One of the best ways to protect and promote the physical and psychosocial health of elderly individuals and provide them with health service is regular home visits. Individuals' needs for health and care increase in old ages. Community health nurses who provide care for elderly individuals in their own environment increase the quality of life of elderly individuals and their families and provide them with healthcare based on clinical care, education and counseling through home visits. Although this subject has such importance, no meta-analysis studies were found to have been conducted on the effect of home visits by nurses on the physical and psychosocial health of elderly individuals in the last 20 years. Studies should be conducted on the effect of home visits by nurses on the physical and psychosocial health of elderly people. PICOS ( participants, interventions, comparisons, outcomes, and study design) used in describing the problem. The objective of this quantitative systematic review and meta analysis is to identify and synthesize the evidence on the effectiveness of home visits by nurses on the physical and psychosocial health of the elderly. The proposed review will aim to address the following questions:

1. What is the effect size of home visits regarding the physical and psychosocial health of the elderly?

2. What is the effect size of the health outcomes of home visits regarding the physical and psychosocial health of the elderly according to gender? Is gender a moderator variable?

3. What is the effect size of the health outcomes of home visits regarding the physical and psychosocial health of the elderly according to country of residence? Is country of residence a moderator variable?

4. What is the effect size of the health outcomes of home visits regarding the physical and psychosocial health of the elderly according to the unit they receive home care services?

5. What is the effect size of the health outcomes of home visits regarding the physical and psychosocial health of the elderly according to the duration and frequency of the visits? Are the duration and frequency of the visits moderator variables?

6. What is the effect size of the health outcomes of home visits regarding the physical and psychosocial health of the elderly according to the status of receiving home based care? Is the status of receiving home based care a moderator variable? 
7. What is the effect size of the health outcomes of home visits regarding the physical and psychosocial health of the elderly according to whether or not using model? Is whether or not using model a moderator variable?

8. What is the effect size of the health outcomes of home visits regarding the physical and psychosocial health of the elderly according to the elderly population studied (general population, those with health problems)?

\section{MATERIAL AND METHODS}

\subsection{Design and methods}

The study carried out August 2016 and May 2017.

\subsection{Types of participants}

1. English and Turkish studies (articles, investigation reports and dissertations) published in refereed and non-refereed journals as well as electronic journals between December, 2004 and December, 2016.

2. Published or unpublished study resources: Master's and $\mathrm{PhD}$ theses

3. Studies with appropriate research method [randomized controlled studies, quasiexperimental studies and observational studies (cohort, case-control, cross-sectional)].

4. Studies with sufficient numeric data for analysis (Pearson's correlation coefficients, chi-square values and t-, p, sample size $(\mathrm{N}), \mathrm{F}$-values, etc. or calculability of these values)

5. Studies including a home visit attempt (practice, intervention, life satisfaction, etc.) to promote the physical (nutrition, hypertension, physical activity, cancer, stroke, pain, falling, sleep, etc.) or psychosocial (dementia, depression, quality of life, social support, loneliness, social isolation, anxiety, life satisfaction, etc.) health of the elderly

6. Studies in which the intervention is carried out by home visits (at least one step of the intervention )

7. Home-based practices performed via home visits,

8. Home visits are carried out by nurses, or nurses direct those carrying out the home visits,

9. The studies design of randomized controlled, quasi-experimental and observational (cohort, case-control, cross-sectional)

10. Quantative studies having received a "moderate" or "strong" score on the quality assessment tool.

\subsection{Types of interventions}

This systematic review and meta analysis will consider studies that effect of home visit interventions by nurses on the elderly health. Health outcomes will include physical (nutrition, hypertension, physical activity, cancer, stroke, pain, falling, sleep, etc.) and psychosocial (dementia, depression, quality of life, social support, loneliness, social isolation, anxiety, life satisfaction, etc.) health. Interventions may include face-to-face delivery, group delivery or any other relevant method or combination of methods in participants' homes. The interventions may involve one or more sessions and can also vary in duration. It is carried out by home visits, or home-based practices performed via home visits. A nurse is a person who is legally licensed and/or registered to practice the full scope of nursing in his or her country.

\subsection{Types of outcomes}

Primary outcomes: quality of life, social support, life satisfaction, mortality, falling, depression, management of hypertension, pain, social isolation Secondary outcomes: SelfReported perceived health, emergency department visits, length of hospital stay. 


\subsection{Types of studies}

The randomized controlled studies, quasi-experimental studies and observational studies (cohort, case-control, cross-sectional).

\section{Exclusion criteria}

1. Studies written in other languages than Turkish and English

2. Visits carried out by another health professional than nurses

3. Studies including insufficient numeric data for analysis

4. Duplicates: for the studies published in more than one paper, the paper with largest sample size will be included.

5. Studies whose full data will not be accessible even after requested from the authors.

6. Studies having received a poor score on the quality assessment tool

\subsection{Search strategy}

\section{Search Process (keywords)}

Meta analysis, home visits, (in-home visiting, domiciliary visits), nursing, elderly health, community health nursing, health visitors, visiting nurses, physical health, psychosocial health, old people, aged, elderly people, elders, seniors, intervention, effect, etc. and various combinations of their Turkish equivalents were used during the search. We were benefited MeSH (Medical Subject Headings) terms for selection of key words. Medline (6860 articles), Web of Sciences (1933 articles), Pubmed (1551 articles), Science Direct (1021 articles), Cinahl Plus with Full Text (1020 articles), Cochrane (501 articles) Tubitak (98 articles), and Yök (126 articles) were searched among a total of 13.110 abstract databases. This search was completed in six months. The search was limited to the studies conducted on home visits by nurses in the last 11 years (between December, 2004 and December, 2016), conducted in Turkish and English, and conducted only with the elderly population. However, advance search will continue until the end of the study.

The first stage of this systematic review will involve the evaluation of titles, abstracts and eligibility of studies. In the second stage, full text of the papers will be independently assessed by two independent observers to confirm their eligibility. Bibliographic details of the studies will be downloaded or manually entered into the EndNote X747 references management database. The search for ongoing clinical trials will be performed in the following databases: www. controlled - trials. com; www. clinical trials. com; www.who.int/trialsearch. Manuel searches will be conducted in key journals. Government reports, theses and dissertations and their references lists.

\subsection{Assessment of methodological quality}

Quality assessments (risk of bias assessments) will be conducted by two independent reviewers for each study included. The Quality Assessment Tool for Quantitative Studies, developed by academic members in master's program within the scope of the Effective Public Health Practice Project (EPHPP) will be used for quality assessment. It is a valid and reliable tool (Thomas et al., 2004). This tool consists of eight sections: bias of selection, study design, confounder, blinding, data collection method, exclusion from and withdrawal from the study, integrity of intervention, and analysis. Except for integrity of intervention and analysis, each section is scored as $1=$ Strong, 2=Moderate, and 3=Poor. After each section is scored, the study is given a general score based on the dictionary. Having no Poor scores indicates a methodologically strong study, one Poor score indicates a study with moderate reliability, and two or more Poor scores indicates a methodologically unreliable study. Based on the assessment and scoring, each assessor expresses their final decision as 1=Strong, 2=Moderate, 
and $3=$ Poor. After scoring, any inconsistencies between the assessors are examined along with the reasons for any inconsistency. No scores are given for intervention integrity and analysis. These sections guide the assessors when they have hesitation about the quality of the study, as well as contributing to the Discussion section of this study. The formation of the Turkish version and validity reliability study have been conducted by researchers. Permission has been obtained from the relevant institution for its use. The validity and reliability tests are conducted for its Turkish adaptation. The expert evaluation showed a content validity index was 0.99. The opinions of eight experts were evaluated using Kendall W analysis, which revealed that there was no statistical difference (Kendall $\mathrm{W}=0.13, \mathrm{p}=0.319$ ) among their opinions and that their scores were consistent with each other. It is a reliable tool that can be used to assess the quality of quantitative studies.

This protocol adheres to PRISMA-P standards for protocol reporting. And we are using flow diagram. We shall conduct a systematic review and meta-analysis and we will report the outcomes according to the Preferred Reporting Items for Systematic Reviews and Meta-Analyses (PRISMA) statement The PRISMA-P checklist contains 17 items considered to be essential and minimum components of a systematic review or meta-analysis protocol (Moher et al., 2009).

This protocol was registered with the International Prospective Register of Systematic Reviews (PROSPERO) on 05-0-2017. PROSPERO 2017: CRD42017054228 Available https://www.crd.york.ac.uk/PROSPERO/display_record.asp?ID=CRD4201705422 8. This study is supported by Selçuk University Scientific Research Projects Coordinator (Project No: 15102040). And approval was obtained from the Ethics Committee of Selcuk University.

\subsection{Data extraction}

The study titles and abstracts will be screened by two authors in the review group independently to identify eligible studies. The articles selected by two independent observers will be compared and a consensus will be made by discussing on the different ideas regarding an article. Two independent observers who have worked on research and elderly health will ensure the reliability of the coding. The study coding protocol including study number, title, authors, year of the study, country of the study, publication type, status of being published, content, objectives, status of receiving institutional services, elderly population studied, status of whether or not using model, the frequency and duration of the visits, study design, sample size, dependent and independent variables analyzed in the study, calculated measures and statistical methods will be used for coding. Inter-coders reliability will be sought by Kappa analysis.

\subsection{Data synthesis}

The methods appropriate for the data type among the methods of integrating the study results will be selected for data analysis. Analyses will be performed using the Comprehensive Meta-Analysis (CMA) version 3 program to apply the meta analysis technique. The studies will be assessed for statistical heterogeneity considering the settings, populations, interventions and outcomes. The statistical heterogeneity of the combined studies will be tested using the I-squared, which describes the percentage of total variation across a study due to heterogeneity rather than chance. Publication bias will also be assessed using a funnel plot, Orwin's failsafe $N$ test, and Duval and Tweedie's trim and fill tests.

\section{RESULTS}

Data collection is ongoing. At the same time 13.110 abstract were screened. Quality assessment is continuing. 


\section{DISCUSSION AND CONCLUSION}

Scientific evidence exists for the fact that more frequent home visits reduce mortality and hospitalization. However, studies analyzing the effectiveness of home visit programs for elderly people may have conflicting results. Researchers have reported that a single study cannot be adequate when conflicting results are seen in the solution of a problem and recommended that the results of independent studies on the same subject be synthesized. Systematics reviews and meta analysis will provide the highest level of informed decisions. Despite there are many meta-analyzes in this issue, there are no data that it had been in the last 20 year and so comprehensive in the literature. But one limitation of this study is that the studies conducted on home visits by nurses in the last 11 years (between December, 2004 and December, 2016), conducted in Turkish and English, and conducted only with the elderly population. But we hope that it will lead to future works.

\section{Acknowledgment}

This study has been derived from doctoral thesis (Emine ERGIN) supported by Selcuk University Scientific Research Projects Coordinator (Project No: 15102040). Researcher has consulted Professor Doctor Emel Ege, Associate Professor Doctor Filiz Hisar, Assistant Professor Dilek Cingil in the period of language and content validity and reliability analysis, and thanks them for their valuable time and effort. And this protocol was registered with the International Prospective Register of Systematic Reviews (PROSPERO) on 05-0-2017. PROSPERO 2017: CRD42017054228 Available https://www. crd.york. ac.uk/ PROSPERO /display_record.asp? ID= CRD42017054228

\section{Ethics}

Study was approved by the Ethics Committee of Selcuk University (ECSU), ECSU No: 2015/48, 30.06.2015.

\section{Conflicts of interest}

Authors declare that there are no conflicts of interest. 
ERGIN / The Effect of Home Visits by Nurses on the Physical and Psychosocial Health of the Elderly: Study Protocol for A Systematic Review and Meta-Analysis

\section{REFERENCES}

BORENSTEIN M., HEDGES L.V., HIGGINS J., 2009, Introduction to Meta-Analysis. John Wiley \& Sons Inc, Hoboken, NewJersey, p; 5.

CHATTERJI S., BYLES J., CUTLER D., 2015. Health, functioning, and disability in older adults-present status and future implications. Lancet, 385: (9967) 563-75.

GRADY P.A., 2011. Advancing the health of our aging population: A lead role for nursing science, Nurs Outlook, 59:207-9.

GILLESPIE L.D., ROBERTSON M.C., GILLESPIE W.J., 2009. Interventions for preventing falls in older people living in the community. Cochrane Database Syst Rev, 15:CD007146.

HAIDICH A.B., 2010. Meta-analysis in medical research. Hippokratia, 14:29-37.

MOHER D., LIBERATI A.,TETZLAFF J., 2009. The PRISMA Group 2009: Preferred reporting items for systematic reviews and meta-analyses: The PRISMA Statement. BMJ , 339: b2535.

STEPTOE A., DEATON A., STONE A.A., 2015. Subjective well being, health, and ageing, Ageing 4. Lancet, 385(9968): 640-48.

STUCK A.E., EGGER M., HAMMER A., 2002. Home visits to prevent nursing home admission and functional decline in elderly people: systematicreviewand metaregression analysis. JAMA,287:1022-28.

SUZMAN R., BEARD J.R., BOERMA T., 2015. Health in an ageingworld - what do we know? Lancet, 385: 484-86.

TARRICONE R., TSOUROS A., 2008. Home care in Europe: the solid facts. Copenhagen, WHO Regional Office for Europe, p.1-33.

TEZCAN S., SEÇKINNER P., 2012, Demographic change in Turkey: aging perspective. Palme Publishing: Ankara.

THOMAS B.H., CILISKA D., DOBBINS M., 2004. A process for systematically reviewing the literature: providing the research evidence for public health nursing interventions. WORLDV EVID-BASED NU, 1:176-84.

VAN HAASTREGT J.C., DIEDERIKS J.P.,VAN ROSSUM E., 2000. Effects of preventive home visits to elderly people living in the community: systematic review. BMJ, 320: 754-58.

WORLD POPULATION AGEING (WPA), 2015. United Nations Department of Economic and Social Affairs. United Nations: WHO. Avaible from: http:// www.un.org /en/development/desa/ population/theme /ageing/. Accessed on: December 2017.

WORLD POPULATION PROSPECTS (WPP) Revision, 2012. United Nations Department of Economic and Social Affairs.Avaible from: http:// esa.un.org/ unpd/wpp/publications /Files/WPP2012_ HIGH-LIGHTS.pdf. Accessed on: December 2017. 